\title{
The effects of ellagic acid on the liver and remote organs' oxidative stress and structure after hepatic ischemia reperfusion injury caused by pringle maneuver in rats
}

\author{
Kapan $\mathrm{M}^{1}$, Gumus $\mathrm{M}^{1}$, Onder $\mathrm{A}^{1}$, Firat $\mathrm{U}^{2}$, Kemal Basarali $\mathrm{M}^{3}$, Boyuk $\mathrm{A}^{1}$, \\ Aliosmanoglu $\mathrm{I}^{1}$, Buyukbas $\mathrm{S}^{3}$ \\ Department of General Surgery, Dicle University Medical Faculty, Diyarbakır, Turkey. \\ drmuratkapan@gmail.com
}

\begin{abstract}
Introduction: We aimed to investigate the possible protective effects of ellagic acid (EA) on the liver and remote organs against the hepatic ischemia-reperfusion injury.

Methods: Forty Wistar-Albino rats were divided into four groups each containing 10 rats. Group I with laparotomy only, Group II with laparatomy and ellagic acid application, Group III with hepatic ischemia-reperfusion and Group IV with hepatic ischemia-reperfusion and ellagic acid application. Hepatic ischemia was induced by pringle's manoeuvre for 30 minutes followed by 30 minutes reperfusion period. After induction of ischemia, EA was applied via oral gavage at a dose of $85 \mathrm{mg} / \mathrm{kg}$. Blood samples were taken from the animals for biochemical analysis at $60^{\text {th }}$ minute of the experiment in all groups. Simultaneously, liver, lung and kidney tissues were sampled for biochemical analyses and histopathological examinations.

Results: The administration of EA reduced serum malonyldialdehid levels $(p<0.05)$ and liver's oxidative stress index compared with the non-use EA groups $(p<0.05)$. Although, ameliorated liver histopathological changes were seen in EA groups, these changes were not statistically significant $(p>0.05)$. The use of EA did not exert significant protective effects against the effects of liver ischemia-reperfusion injury on the kidney and lung. Conclusion: In our experiments ellagic acid reduced the liver oxidative stress induced by ischemia-reperfusion injury. However, no significant histological improvement was found with EA. There were no significant protective effects on the remote organ injuries induced by ischemia-reperfusion (Tab. 3, Fig. 7, Ref. 37). Full Text in PDF www.elis.sk.

Key words: hepatic ischemia reperfusion, ellagic acid, liver, remote organs.
\end{abstract}

\begin{abstract}
Abbreviations: ischemia reperfusion - IR, reactive oxygen species - ROS, ellagic acid - EA, thiobarbituric acid - TBA, total antioxidant capacity - TAC, malonyldialdehid - MDA, total oxidant activity - TOA, oxidative stress index - OSI
\end{abstract}

The intermittent portal triad clamping technique called Pringle's manoeuvre is an effective procedure for control of blood loss from the hepatic parenchyma during hepatic surgery. However, occlusion of hepatic blood flow, depending on the process, leads to hepatic ischemia reperfusion (IR) injury (1). Hepatic IR injury also may occur due to trauma, sepsis and liver transplantation (2). The lack of oxygenation of the liver leads to serious damage in the liver during ischemia. However, the actual damage is created by reactive oxygen species (ROS) during reperfusion period (3). Reactive oxygen species may initiate oxidative stress by causing

${ }^{1}$ Department of General Surgery, Dicle University Medical Faculty, Diyarbakır, Turkey, ${ }^{2}$ Department of Biochemistry, Dicle University Medical Faculty, Diyarbakır, Turkey, and ${ }^{3}$ Department of Pathology, Dicle University Medical Faculty, Diyarbakır, Turkey.

Address for correspondence: M. Kapan, MD, Dicle Universitesi, Tip Fakültesi, Genel Cerrahi AD, 21280, Diyarbakir, Turkey.

Phone: +90.412.248.8001/4153, Fax: +90.412.248.8523. lipid peroxidation (4) and ROS cause inflammatory response and tissue damage by activating some mediators. Also, ROS directly leads to harmful effects on the cell components (2). Therefore, scavengers of ROS and antioxidant substances are thought to be beneficial in hepatic ischemia reperfusion injury (3).

Flavonoids are polyphenolic antioxidants and naturally found in vegetables and fruits (5). It has been reported that plant based polyphenolic compounds have anti-inflammatory, antimutagenic, anticarcinogenic, and antiviral activity (6). Ellagic acid (EA) is a phenolic component of some fruits and nuts, such as raspberries, strawberries, mango kernel, longan seed, walnuts and pomegranate (5). EA indicates antioxidant effect by preventing lipid peroxidation and protects the cells from oxidative damage (6). It has been reported that EA alters enzyme activities such as glutathione peroxidase, catalase, quinone reductase and glutathione-S-transferase (7). EA shows a strong antioxidant activity with it's directantioxidant activity and reduction of the ROS induced depletion of glutathione peroxidase and catalase activities (5).

In this paper, we aimed to investigate the protective effects of EA on the liver and remote organs against the hepatic IR injury. We analyzed the alterations in the oxidant and antioxidant status by measuring total antioxidant capacity (TAC), malonyldialdehid 
(MDA) in the plasma and TAC, total oxidant activity (TOA) and oxidative stress index (OSI) in the liver, kidney and lung. We also examined histopathological changes in the liver, kidney and lung.

\section{Materials and methods}

\section{Experimental animals}

Forty male Wistar albino rats with mean weight of 250 to 300 grams were included into study at Dicle University Health Sciences Application and Research Center. The study protocol was approved by the Committee of Experimental Animals of Dicle University. All experimental procedures complied with the Guide for the Care and Use of Laboratory Animals. Rats were housed in an air-conditioned room with $12 \mathrm{~h}$ light and dark cycles, with constant temperature $\left(22 \pm 2{ }^{\circ} \mathrm{C}\right)$. The rats were housed in cages, and allowed free access to standard rat chow and water before the experiments. The animals were fasted overnight before the experiments, but were enabled free access to water.

\section{Experimental protocol}

Rats were anesthetized with $50 \mathrm{mg} / \mathrm{kg}$ ketamine hydrochloride (Ketalar ${ }^{\circledR}$, Parke Davis, Eczacibasi, Istanbul, Turkey) and $10 \mathrm{mg}$ kg xylazine (Rompun ${ }^{\circledR}$, Bayer AG, Leverkusen, Germany) via intramuscular injection and experimental procedure was initiated. A $10 \%$ povidone iodine solution (Betadine $\left({ }^{\circledR}\right)$ was applied for shaved skin cleansing. A midline incision was performed and rats underwent either sham surgery or ischemia-reperfusion. Ischemia was induced with Pringle's manoeuvre and sustained for $30 \mathrm{~min}$ utes. Thirty minutes later, the ischemic liver was reperfused for 30 minutes. At the end of this period the animals were sacrificed by taking blood from the heart.

Animals were divided into four groups;

Group 1 (Sham): Only hepatoduedonal ligament dissection was performed and no drug was given.

Group 2 (Control): Hepatoduedonal ligament dissection was performed and EA was given at a dose of $85 \mathrm{mg} / \mathrm{kg}$ via oral gavage (according to Tmax), at the beginning of the experimental study (8),

Group 3 (IR): Thirty minutes after Pringle maneuver, reperfusion was constituted 30 minutes and no drug was given.

Group $4(I R+E A)$ : Thirty minutes after Pringle maneuver, reperfusion was generated for 30 minutes and EA was given at a dose of $85 \mathrm{mg} / \mathrm{kg}$ via oral gavage (according to Tmax), after initiation of hepatic ishemia (8).

At the end of the procedures, blood and tissue samples from the liver, lung and kidney were obtained for biochemical analyses and histopathological examinations. Serum was obtained from blood centrifugation and rapidly transferred to plastic ependorf covered tubes for biochemical analyses and stored at $-80^{\circ} \mathrm{C}$ in deep freezer. Taken tissues were prepared for biochemical analyses, foreign tissue residues and blood were removed by washing with saline and thereafter tissues were transferred to plastic tubes with ependorf cover for biochemical analyses, stored at $-80{ }^{\circ} \mathrm{C}$ in a freezer. In addition, the tissues taken for histopathological evaluation were put into plastic containerswith $10 \%$ formaldehyde solution.

\section{Biochemical analyses}

Total antioxidant capacity (TAC) and malondialdehyde (MDA) analyses were performed in blood samples. Total oxidant status (TOA) and TAC analysis were performed in tissue samples. In addition, oxidative stress index (OSI) was calculated in tissue samples.

\section{Homogenization of tissues:}

Tissues stored at $-80^{\circ} \mathrm{C}$ were removed on the deep freezer and brought to the laboratory in dry ice. About 0.30 to 0.50 grams of tissue pieces were transferred into the tube and $2 \mathrm{ml}$ of Tris- $\mathrm{HCl}$ buffer were added. Tissues in the tube were placed into ice-filled plastic container and processed in the $50 \mathrm{mM} \mathrm{pH} 7.0$ phosphate buffer (PBS) for 1-3 minutes on 14,000 rpm at homogenizer (U1tra Turrax Type T8, IKA Labortechnic, Germany). Homogenate was centrifuged for 30 minutes at $+40{ }^{\circ} \mathrm{C}$. Samples were obtained from supernatant for TOA and TAC analysis.

\section{Measurement of malondialdehyde}

MDA levels were estimated by the double heating method of Draper and Hadley (9). The principle of the method is spectrophotometric measurement of the color generated by the reaction of thiobarbituric acid (TBA) with MDA. For this purpose, $2.5 \mathrm{ml}$ of trichloroacetic acid solution ( $10 \%$ ) was added to $0.5 \mathrm{ml}$ plasma in each centrifuge tube, and the tubes were placed in a boiling water bath for $15 \mathrm{~min}$. After cooling in tap water, the tubes were centrifuged at $1000 \mathrm{~g}$ for $10 \mathrm{~min}$, and $2 \mathrm{ml}$ of supernatant were added to $1 \mathrm{ml}$ of TBA solution $(6.7 \mathrm{~g} / \mathrm{L})$ in a test tube, and the tube was placed in a boiling water bath for $15 \mathrm{~min}$. The solution was then cooled in tap water and its absorbance was measured using a spectrophotometer (Shimadzu UV-1208, Japan) at $532 \mathrm{~nm}$. The concentration of MDA was calculated by the absorbance coefficient of the MDA-TBA complex (absorbance coefficient of 1.56x105 $\mathrm{cm}^{-1} \mathrm{M}^{-1}$ ) and is expressed as $\mu \mathrm{mol} / \mathrm{L}$.

\section{Measurement of TOA}

TOA of supernatant fractions was determined using a novel automated measurement method, developed by Erel (10). Oxidants present in the sample oxidize the ferrous ion-o-dianisidine complex to ferric ion. The oxidation reaction is enhanced by glycerol molecules, which are abundantly present in the reaction medium. The ferric ion makes a colored complex with xylenol orange in an acidic medium. The color intensity, which can be measured spectrophotometrically, is related to the total amount of oxidized molecules present in the sample. The assay is calibrated with

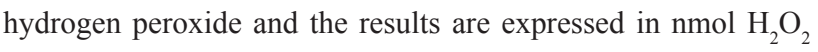
Equiv./mg protein.

\section{Measurement of the TAC}

TAC of supernatant fractions was determined using a novel automated measurement method developed by Erel $(10,11)$. In this method, hydroxyl radical, which is the most potent biological radical, is produced. In the assay, ferrous ion solution, which is present in Reagent 1, is mixed with hydrogen peroxide, which is present in Reagent 2 . The sequentially produced radicals such 
as brown colored dianisidinyl radical cation, produced by the hydroxyl radical, are also potent radicals. Using this method, antioxidative effect of the sample against the potent-free radical reactions, which is initiated by the produced hydroxyl radical, is measured. The assay has excellent precision values, lower than 3 $\%$. The results are expressed as nmol Trolox Equiv./mg protein.

\section{Oxidative stress index}

OSI is an indicator of the degree of oxidative stress, formulation is as follows:

OSI $=[$ TOA(nmol H2O2 Equiv./mg)/TAC(nmol Trolox Equiv./mg)]x100 (12).

\section{Histopathological assessment}

Liver, kidney and lung tissues were put into $10 \%$ formalin solution in paraffin blocks and prepared by slicing $4-\mu \mathrm{m}$ sections.
Tissues stained with hematoxylin-eosin and standard protocols were applied.

Hepatic injury was evaluated for severity using an ordinal scale as follows: Grade 0, minimal damage or no damage; Grade 1 , mild damage with cytoplasmic vacuolization and nuclear pycnosis; Grade 2, moderate damage with expanded nuclear pycnosis, cytoplasmic hypereosinophilia and loss of intercellular borders; Grade 3, severe damage with hemorrhage, neutrophil infiltration and severe necrosis with disintegration of the hepatic adheres (13).

Renal injury was graded as follows: Grade 0, no changes; Grade 1, swelling of tubular cells, loosing of brush edges, nuclear condensation which is showing nuclear looses consisting of onethird of tubular structures; Grade 2, addition to grade 1, nuclear looses ranging from two-thirds of tubular structures; Grade 3, changes including nuclear looses which effect more than twothirds of tubular structures (14).
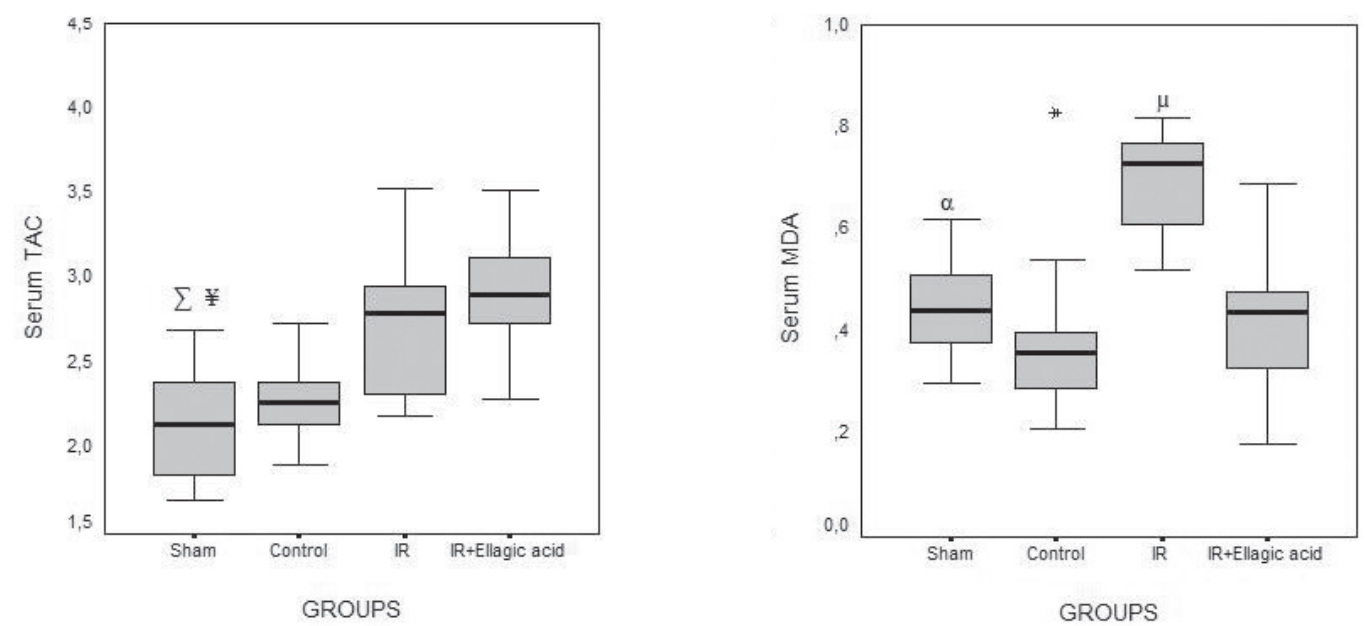

Fig. 1. Serum TAC and MDA Levels according to the groups (IR=Ischemia-reperfusion, MDA= Malondialdehyde, TAC $=$ Total antioxidant capacity. ${ }^{\Sigma} \mathbf{P}=0.002$ Sham vs IR; ${ }^{*} \mathbf{P}<0.001$ Sham vs IR + Ellagic acid; ${ }^{\alpha} \mathbf{p}<0.001$ Sham vs IR; ${ }^{\mu} p<0.001$ IR vs IR + Ellagic acid.)
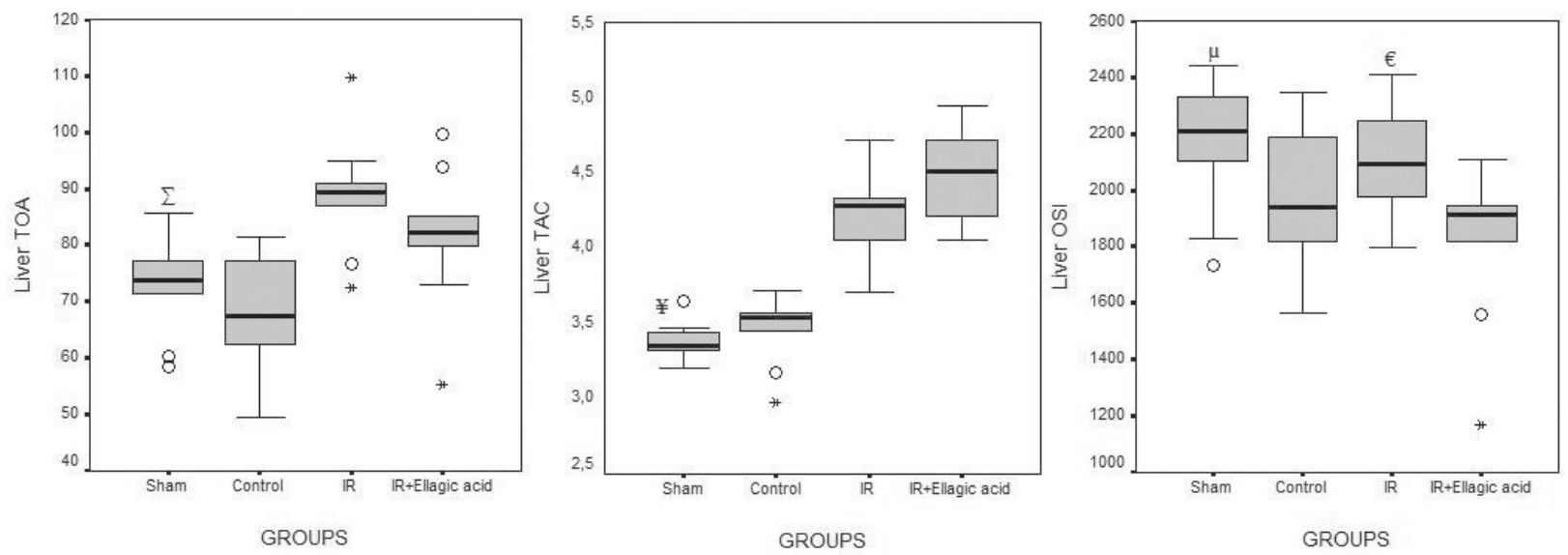

Fig. 2. Liver TOA, TAC and OSI levels according to the groups. (IR=Ischemia-reperfusion, TOA=Total oxidant activity, TAC $=$ Total antioxidant capacity, OSI=Oxidative stress index. ${ }^{\Sigma} p=0.002$ Sham vs IR; ${ }^{x} p<0.001$ Sham vs IR and IR + Ellagic acid; ${ }^{\mu} p=0.007$ Sham vs IR; ${ }^{\epsilon} p=0.010$ IR vs IR + Ellagic acid.) 

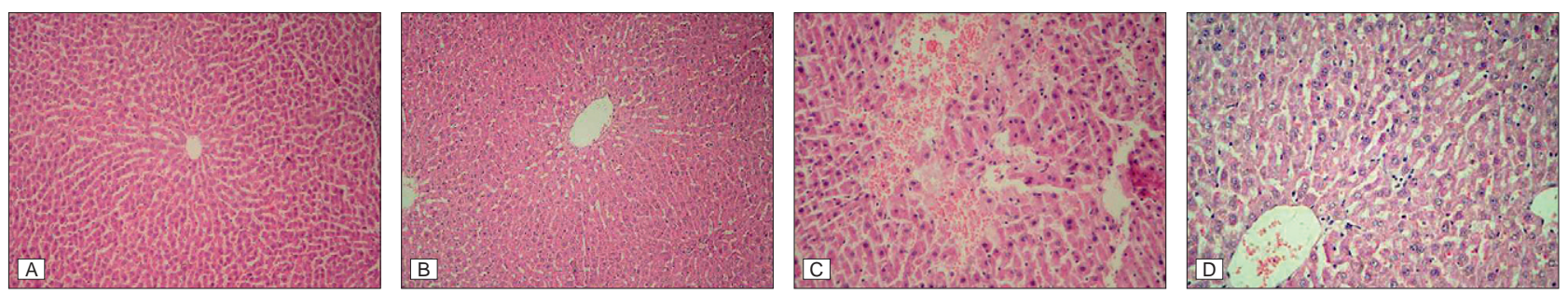

Fig. 3. Effects of EA on hepatic tissue injury after IR evaluated by histological examination. A: In groups Sham, Minimal cellular changes with mild stoplasmic eosinophilia in only a few hepatocytes (H\&E stain,x100); B: In group Control, Almost normal architecture of the liver tissue (H\&E stain,x100); C: In group IR, Cytoplasmic hypereosinophilic changes, nuclear pyknosis, loss of intercellular borders, necrobiosis and necrosis in many hepatocytes can be seen (H\&E stain, x200); D: In group IR+Ellagic acid, Cytoplasmic hypereosinophilic changes, nuclear pyknosis, loss of intercellular borders and necrobiosis in some hepatocytes and haemorrhage in a few disordered areas in the liver tissue are seen (H\&E stain, $\mathbf{x 2 0 0 )}$.
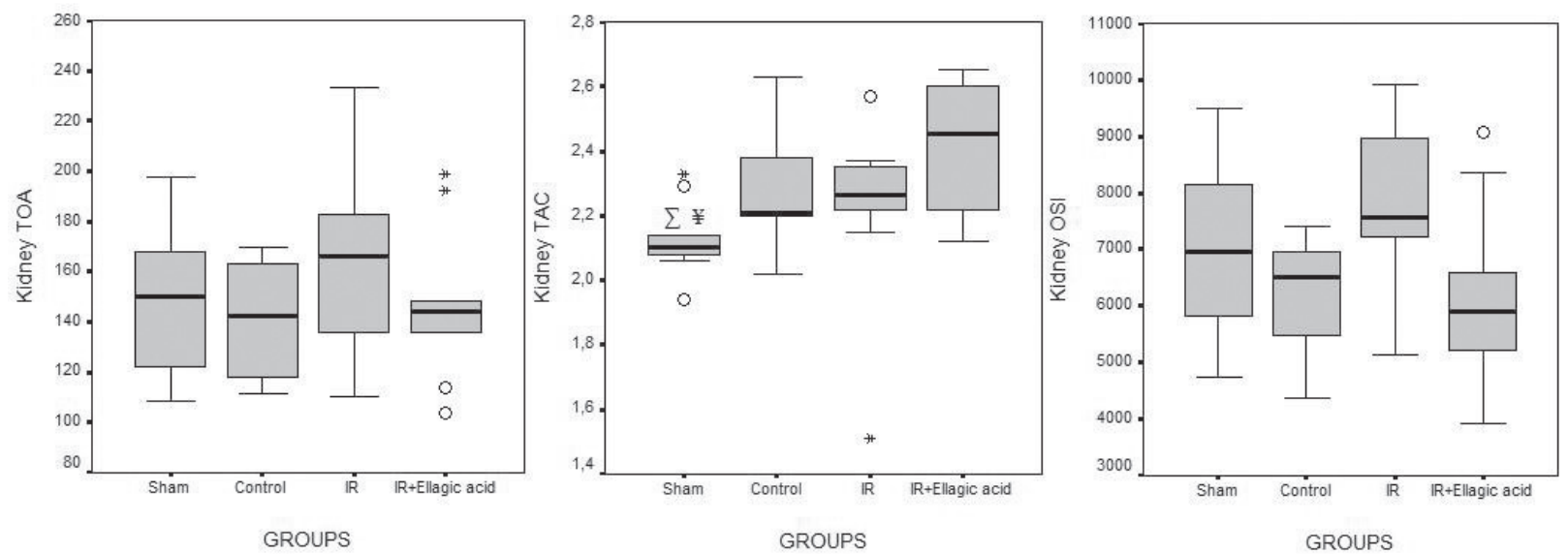

Fig. 4. Kidney TOA, TAC and OSI levels according to the groups. (IR=Ischemia-reperfusion, TOA=Total oxidant activity, TAC=Total antioxidant capacity, OSI=Oxidative stress index. ${ }^{\Sigma} \mathbf{p}=0.023$ Sham vs Control and IR; ${ }^{\mathrm{x}} \mathrm{p}=0.001$ Sham vs IR + Ellagic acid.)
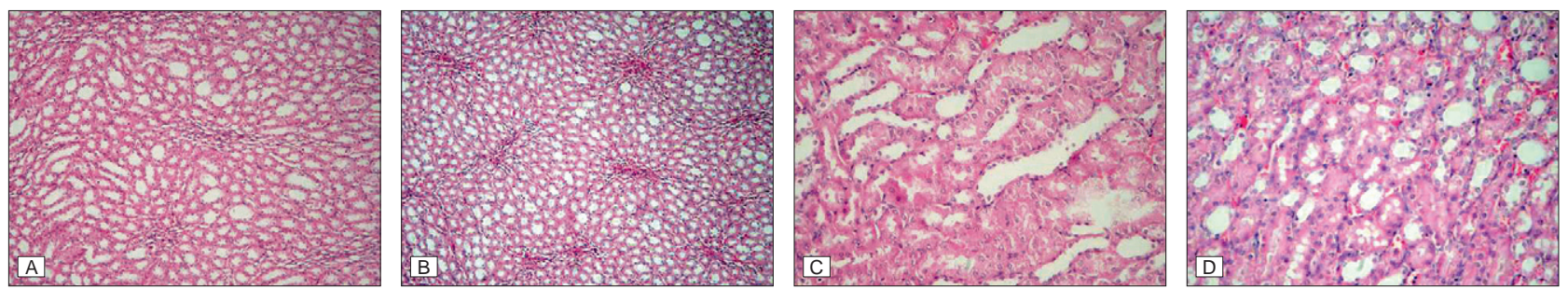

Fig. 5. Effects of EA on kidney tissue injury after IR evaluated by histological examination. A: In group Sham, Tubular cell swelling in few areas (H\&E stain,x100); B: In group Control, Normal morphological architecture in renal tubules (H\&E stain, x200); C: In group IR, Many tubular cells show nuclear loss and cell swelling (H\&E stain,x200); D: In group IR+Ellagic acid, Nuclear loss and cell swelling in some tubular cells; (H\&E stain,x200).

Lung injury was evaluated using an ordinal scale as follows Grade 0 , no damage; Grade 1, mild neutrophil leukocyte infiltration and mild-moderate interstitial congestion; Grade 2, moderate neutrophil leukocyte infiltration, perivascular edema formation and disintegration of the pulmonary structure; Grade 3, dense neutrophil leukocyte infiltration and absolute destruction of pulmonary structure (15).

\section{Statistical analysis}

Statistical analysis was performed by SPSS for Windows 11.5 (SPSS Inc., Chicago, IL, USA). Data were presented as mean (minimum, maximum) values for biochemical values. Groups were compared by using the nonparametric Kruskal-Wallis test. MannWhitney U test was used for binary comparisons. Spearman correlation test was used for evaluation of the relationships of the numerical variables. $P$ value of less than 0.05 were considered significant.

\section{Results}

Effects of EA on serum MDA and TAC levels and liver TAC, TOA, OSI levels and histopathological scores induced by hepatic IR

All of the parameters, except OSI, between the sham group 


\section{$274-281$}
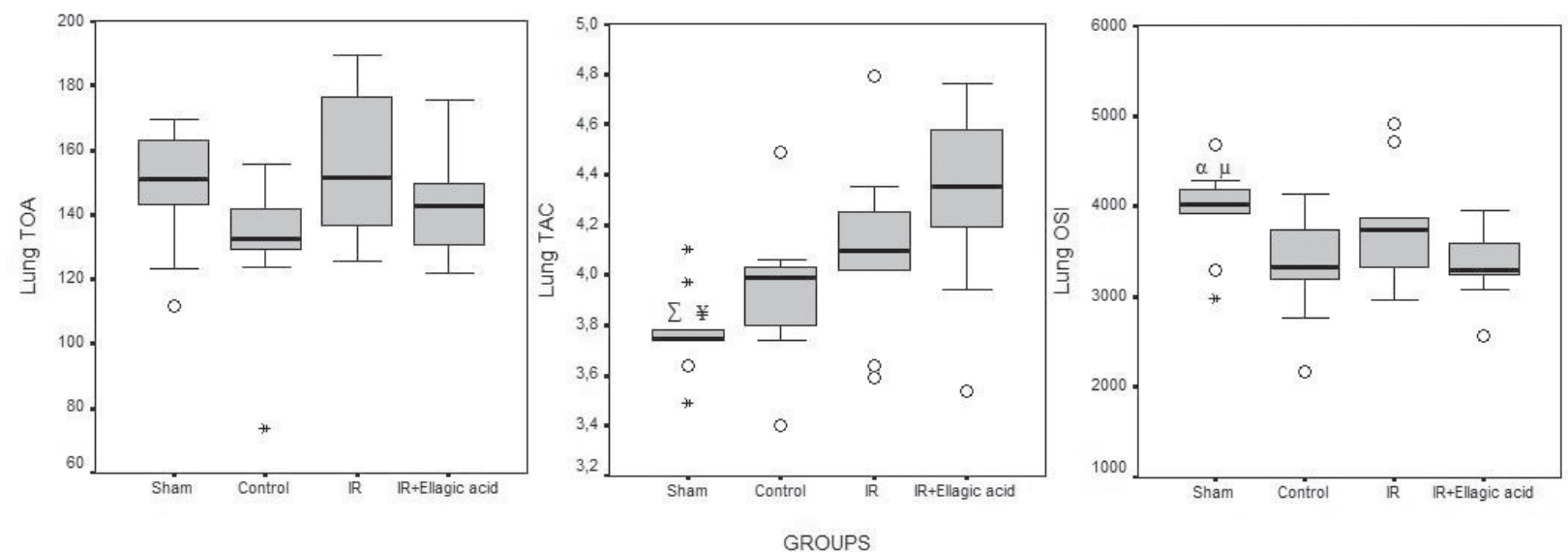

Fig. 6. Lung TOA, TAC and OSI levels according to the groups. (IR=Ischemia-reperfusion, TOA=Total oxidant activity, TAC $=$ Total antioxidant capacity, OSI=Oxidative stress index. ${ }^{\Sigma} p=0.029$ Sham vs IR; ${ }^{*} p=0.002$ Sham vs IR + Ellagic acid; ${ }^{\alpha} p=0.019$ Sham vs Control; ${ }^{\mu} p=0.011$ Sham vs IR + Ellagic acid).
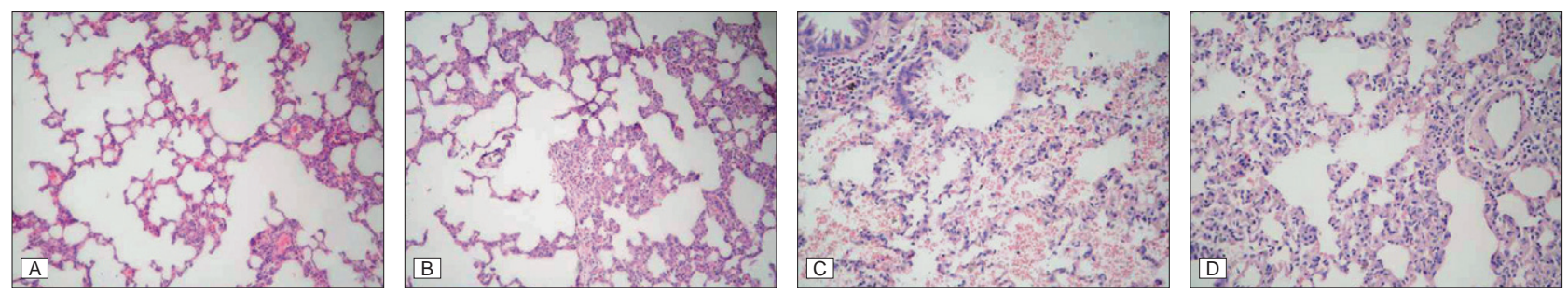

Fig. 7. Effects of EA on lung tissue injury after IR evaluated by histological examination. A: In group Sham, Mild PNL infiltration and mild interstitial congestion in the lung tissue (H\&E stain,x100); B: In group Control, Moderate interstitial congestion with minimal inflammation (H\&E stain,x100); C: In group IR, Neutrophil leukocyte infiltration and perivascular edema with disintegration of the structure in the tissue (H\&E stain,x200); D: In group IR+Ellagic acid, Moderate PNL infiltration and interstitial congestion in the lung tissue are seen (H\&E stain, $x 200)$.

and IR group were found to be significantly different $(\mathrm{p}=0.002$ for serum TAC and liver TOA, $\mathrm{p}<0.001$ for serum MDA, liver TAC and histopathological scores). In IR group significantly increased serum MDA and TAC levels, and liver TAC and TOA levels and severe liver injury were found. EA significantly reduced serum MDA levels ( $<<0.001)$ (Fig. 1). Liver's OSI levels were found to be significantly lower in EA-used groups compared with the non-use EA groups $(\mathrm{p}<0.05)$ (Fig. 2). Although some histological ameliorations were found in EA groups, these histopathological changes were not reached to a statistically significant level ( $>00.05)$ (Fig. 3). EA had no effect on other parameters (Table 1$)$.
Effects of EA on kidney TAC, TOA, OSI levels and histopathological scores induced by hepatic IR

IR significantly increased kidney TAC levels and led to severe kidney injury, but achieved no significant change on kidney TOA and OSI levels (Figs 4 and 5). Administration of EA did not lead to significant changes of all parameters in the kidney (Tab. 2).

Effects of EA on lung TAC, TOA, OSI levels and histopathological scores induced by hepatic IR

Increased lung TAC levels and moderate lung injury were observed after hepatic IR. But there were no significant changes in lung TOA and OSI levels following IR (Figs 6 and 7). Ad-

Tab. 1. Serum and liver findings of the study groups.

\begin{tabular}{|c|c|c|c|c|c|}
\hline & $\begin{array}{c}\text { Sham } \\
(\text { Mean } \pm \text { SD) }\end{array}$ & $\begin{array}{c}\text { Control } \\
(\text { Mean } \pm \text { SD })\end{array}$ & $\begin{array}{c}\text { IR } \\
(\text { Mean } \pm \text { SD) }\end{array}$ & $\begin{array}{l}\text { IR+Ellagic acid } \\
(\text { Mean } \pm \text { SD })\end{array}$ & $\begin{array}{c}\text { Difference } \\
\text { (p) }\end{array}$ \\
\hline Serum TAC ( $\mu$ mol Trolox) Equiv/L) & $2.14 \pm 0.32$ & $2.38 \pm 0.43$ & $2.87 \pm 0.62$ & $2.94 \pm 0.37$ & $<0.001$ \\
\hline $\operatorname{Serum} \operatorname{MDA}(\mu \mathrm{M} / \mathrm{L})$ & $0.44 \pm 0.10$ & $0.40 \pm 0.18$ & $0.69 \pm 0.10$ & $0.44 \pm 0.15$ & 0.001 \\
\hline Liver - TAC (nmol Trolox) Equiv/mg) & $3.38 \pm 0.12$ & $3.46 \pm 0.22$ & $4.2 \pm 0.28$ & $4.49 \pm 0.30$ & $<0.001$ \\
\hline Liver - TOA $\left(\mathrm{nmol} \mathrm{H}_{2} \mathrm{O}_{2}\right)$ Equiv/mg) & $73.17 \pm 8.45$ & $68.33 \pm 9.50$ & $88.87 \pm 10.01$ & $81.62 \pm 11.83$ & $<0.001$ \\
\hline Liver - OSI & $2165.66 \pm 225.34$ & $1974.03 \pm 242.44$ & $2106.20 \pm 195.86$ & $1825.45 \pm 275.32$ & 0.029 \\
\hline Liver - HP & $0.30 \pm 0.48$ & $0.30 \pm 0.48$ & $2.30 \pm 0.48$ & $2.10 \pm 0.74$ & $<0.001$ \\
\hline
\end{tabular}

$\overline{\mathrm{TAC}}$ - total antioxidant capacity, TOA - total oxidant activity, MDA - malonyldialdehid, OSI - oxidative stress index, HP - histopathological score, SD - standard deviation, NS - not significant 
Tab. 2. Kidney findings according in the study groups.

\begin{tabular}{|c|c|c|c|c|c|}
\hline & $\begin{array}{c}\text { Sham } \\
(\text { Mean } \pm \text { SD })\end{array}$ & $\begin{array}{c}\text { Control } \\
(\mathrm{Mean} \pm \mathrm{SD})\end{array}$ & $\begin{array}{c}\text { IR } \\
(\text { Mean } \pm \text { SD })\end{array}$ & $\begin{array}{c}\mathrm{IR}+\mathrm{EA} \\
(\mathrm{Mean} \pm \mathrm{SD})\end{array}$ & $\begin{array}{c}\text { Difference } \\
\text { (p) }\end{array}$ \\
\hline Kidney - TAC (nmol Tolox Equiv/mg) & $2.12 \pm 0.11$ & $2.29 \pm 0.19$ & $2.23 \pm .28$ & $2.43 \pm 0.19$ & 0.005 \\
\hline Kidney - TOA (nmol $\mathrm{H}_{2} \mathrm{O}_{2}$ Equiv/mg) & $149.38 \pm 30.56$ & $141.83 \pm 22.16$ & $168.95 \pm 39.19$ & $146.48 \pm 29.78$ & NS \\
\hline Kidney - OSI & $7039.21 \pm 1487.97$ & $6217.43 \pm 970.42$ & $7638.72 \pm 1625.39$ & $6120.82 \pm 1599.40$ & NS \\
\hline Kidney - HP & $0.20 \pm 0.42$ & $0.10 \pm 0.31$ & $2.60 \pm 0.69$ & $2.40 \pm 0.70$ & $<0.001$ \\
\hline
\end{tabular}

Tab. 3. Lung findings according to the study groups.

\begin{tabular}{|c|c|c|c|c|c|}
\hline & $\begin{array}{c}\text { Sham } \\
(\text { Mean } \pm \text { SD })\end{array}$ & $\begin{array}{c}\text { Control } \\
(\mathrm{Mean} \pm \mathrm{SD})\end{array}$ & $\begin{array}{c}\text { IR } \\
(\text { Mean } \pm \text { SD })\end{array}$ & $\begin{array}{c}\mathrm{IR}+\mathrm{EA} \\
(\mathrm{Mean} \pm \mathrm{SD})\end{array}$ & $\begin{array}{c}\text { Difference } \\
\text { (p) }\end{array}$ \\
\hline LUNG - TAC (nmol Trolox Equiv/mg) & $3.77 \pm 0.16$ & $3.94 \pm 0.28$ & $4.10 \pm 0.34$ & $4.31 \pm 0.36$ & 0.004 \\
\hline LUNG - TOA (nmol $\mathrm{H}_{2} \mathrm{O}_{2}$ Equiv/mg) & $148.75 \pm 18.74$ & $131.02 \pm 22.72$ & $154.04 \pm 20.86$ & $143.08 \pm 14.94$ & NS \\
\hline LUNG - OSI & $3944.24 \pm 486.39$ & $3319.02 \pm 562.80$ & $3779.81 \pm 624.19$ & $3331.14 \pm 372.79$ & 0.024 \\
\hline LUNG - HP & $0.50 \pm 0.47$ & $0.10 \pm 0.32$ & $1.70 \pm 0.483$ & $1.30 \pm 0.483$ & $<0.001$ \\
\hline
\end{tabular}

$\overline{\mathrm{TAC}}$ - total antioxidant capacity, TOA - total oxidant activity, MDA - malonyldialdehid, OSI -oxidative stress index, HP - histopathological score, SD - standard deviation, NS - not significant

ministration of EA had no effect on all studied variables in the lung (Tab. 3).

\section{Discussion}

Intraoperative bleeding is a major problem during liver resections and the amount of blood loss has been reported as a main factor associated with morbidity and mortality (16). Pringle manoeuvere, continuous or intermittent clamping of the hepatoduedonal ligament, is an effective technique which is used for reducing the hemorrhage (17). But using this technique leads to hepatic IR injury and results in structural and functional disturbances in the liver. This method also induces systemic inflammation $(3,17,18)$. Hepatic IR injury is a complex process accompanied by intracellular signaling pathways, mediators, cells and pathophysiological alterations (3). There are three main mechanisms associated with the pathophysiological alterations of hepatic IR injury: microcirculatory failure, inflammatory response and formation of ROS (19). Especially, the damage is mediated by ROS in the early stage of hepatic IR injury (20). ROS can induce lipid peroxidation which is related to liver damage caused by IR (21). When reaching large amounts, they can lead to direct oxidative damage through ironmediated reactions (22). Therefore, various clinical studies are focused on scavenging and formation of ROS (3).

Due to the effects of scavenging ROS, EA has a strong antioxidant capacity (6). EA inhibits the formation of ROS in both enzymatic and non-enzymatic systems by chelating divalent cations (23). It has been reported that EA prevents cell membrane injury by increasing the levels of non-enzymatic antioxidants (Vitamin $\mathrm{C}$ and Vitamin E) in tissue (6). It has also been reported that EA directly influences the intracellular regulatory mechanisms by the effects on double-stranded DNA (24). Thus, we aimed to investigate the effects of EA on hepatic IR injury together with remote organ IR damage.

Increased levels of MDA are an indicator of lipid peroxidation and membrane disintegration as a result of oxidative damage $(25$,
26). Takayama et al (27) reported that MDA not only reflects the level of ROS, but also can show the severity of liver cell damage. In present study, administration of EA significantly decreased the levels of MDA.

The determination of either oxidants or antioxidants activity can give some information about oxidative stress. However, determination of both of them may be more useful to get information about oxidative stress (28). In the present study, we used TAC which shows antioxidant status and TOA which shows oxidant status. And also we used OSI to evaluate oxidative stress as an indicator of both TAC and TOA (12). In an animal study, it has been reported that administration of EA prevented injury against cyclosporine A-induced oxidative damage in the liver, because of its protective effects on lipid peroxidation and on the endogenous antioxidant systems (6). Yuce et al (5) showed the amelioration of cyclosporine A-induced hepatic damage by EA. Additionally, Hwang et al. (29) demonstrated the protective antioxidant effects of EA. In our study, administration of EA was found ineffective on liver TOA and TAC levels. Only OSI was found as significantly lower in EA groups compared with the non-use EA groups. As a result of hepatic IR injury, cytoplasmic vacuolation, focal nuclear pyknosis and loss of intercellular borders with disintegration of hepatic cords were observed in the study groups and these findings were more common in IR group than IR + EA group.

Hepatic IR injury not only damages the liver, but frequently leads to remote organs' injury such as kidney and lung $(18,30$, $31)$. The development of acute renal failure after major hepatic IR is common $(40-85 \%)$, resulting in high mortality and morbidity in perioperative period $(30,32)$. Davis et al (33) have reported that the pathogenesis of kidney damage associated with hepatic IR can be attributed to the portal hypertension induced splanchnic vasodilation with following intrarenal vasoconstriction. This leads to systemic hypotension, upregulation of renin-angiotensin system and results in damage including, renal tubular necrosis and renal dysfunction. As well other mechanism such as systemic inflammation can lead to the renal damage. However, Polat et 
al.(34) reported that the renal dysfunction was mild with little or no histolopathological alterations. In our study, hepatic IR injury leads to increasing OSI and moderate significant changes of renal morphology. Administration of EA created no significant changes in the kidney morphology compared to hepatic IR.

It has been demonstrated that lung injury can be seen as a result of systemic inflammation initiated after hepatic IR (35). During the early phase of hepatic IR injury, ROS leads to activation of Kupffer cells. After this early phase, the formation of proinflammatory cytokines including TNF- $\alpha$ increases (36). Pulmonary microvascular field is the primary target of these cytokines (35). The increasing levels of TNF- $\alpha$ produce and enhance the pulmonary neutrophil infiltration and microvasculature injury, resulting in lung injury (37). In our study, significant moderate changes were seen in lung morphology after hepatic IR. However, there were no positive effects on the lungs with the administration of EA, as an antioxidant and anti-inflammatory agent. Ota et al (34) demonstrated that ventilation is the main factor of determining lung injury caused by hepatic IR. At the low tidal volume of ventilation, lung injury was not common. Therefore, our findings about the effects of EA on oxidative stress in the lungs can be attributed to the fact that there was no change in ventilation volume between the groups.

\section{Conclusions}

In our study, a significant damage caused by hepatic IR injury caused by pringle manoeuvre was found in liver and remote organs, such as kidney and lung. Ellagic acid reduced the liver oxidative stress from ischemia-reperfusion injury. However, no significant histological improvement was found with EA application. Furthermore, there were no significant effects of EA on the remote organ injuries induced by ischemia-reperfusion.

\section{References}

1. Sugiyama Y, Ishizaki Y, Imamura H, Sugo H, Yoshimoto J, Kawasaki S. Effects of intermittent Pringle's manoeuvre on cirrhotic compared with normal liver. Brit J Surg 2010; 97: 1062-1069.

2. Yildiz F, Coban S, Terzi A, Ates M, Aksoy N, Cakir H, Ocak AR, Bitiren M. Nigella sativa relieves the deleterious effects of ischemia reperfusion injury on liver. World J Gastroenterol 2008; 14: 5204-5209.

3. Şehirli Ö, Özel Y, Dulundu E, Topaloglu U, Ercan F, Şener G. Grape seed extract treatment reduces hepatic ischemia-reperfusion Injury in rats. Phytother Res 2008; 22: 43-48.

4. Gedik E, Girgin S, Öztürk H, Obay BD, Öztürk H, Büyükbayram H. Resveratrol attenuates oxidative stress and histological alterations induced by liver ischemia/reperfusion in rats. World J Gastroenterol 2008; 14: 7101-7106.

5. Yüce A, Atessahin A, Çeribaşı AO. Amelioration of Cyclosporine AInduced Renal, Hepatic and Cardiac Damages by Ellagic Acid in Rats. Basic Clin Pharmacol Toxicol 2008; 103: 186-191.

6. Pari L, Sivasankari R. Effect of ellagic acid on cyclosporine A-induced oxidative damage in the liver of rats. Fundamental Clin Pharmacol 2008; 22: 395-401.
7. Buniatian GH. Stages of activation of hepatic stellate cells: effects of ellagic acid, an inhibiter of liver fibrosis, on their differentiation in culture. Cell Prolif 2003; 36: 307-319.

8. Fan Lei, Dong-Ming Xing, Lan Xiang, Yu-Nan Zhao, Wei Wang, Lu-Jun Zhang, Li-Jun Du. Pharmacokinetic study of ellagic acid in rat after oral administration of pomegranate leaf extract. J Chromatogr B 2003; 796: 189-194.

9. Draper HH, Csallony AS, Hadley N. Urinary aldehydes as indicators of lipid peroxidation in vivo. Free Radic Biol Med 2000; 29: 1071-1077.

10. Erel $\mathbf{O}$. A novel automated method to measure total antioxidant response against potent free radical reactions. Clin Biochem 2004; 37 : $112-119$.

11. Erel O. A new automated colorimetric method for measuring total exident status. Clin Biochem 2005; 38: 1103-1111.

12. Bolukbas C, Bolukbas FF, Horoz M, Aslan M, Celik H, Erel O. Increased oxidative stress associated with the severity of the liver disease in various forms of hepatitis B virus infection. BMC Infect Dis 2005; 5: 95.

13. Kesik V, Güven A, Vurucu S, Tunç T, Uysal B, Gündoğdu G, Öztaş E, Korkmaz A. Melatonin and $1400 \mathrm{~W}$ Ameliorat both intestinal and remote organ infury following mesenteric ishemia/reperfusion. J Surg Res 2009; $1: 1-9$

14. Chatterjee PK, Patel NS, Kvale EO, Cuzzocrea S, Brown PA, Stewart KN, Mota-Filipe H, Thiemermann C. Inhibition of inducible nitric oxide synthase reduces renal ischemia/reperfusion injury. Kidney Int 2002; 61: 862-871.

15. Koksel O, Yildirim C, Cinel L, Tamer L, Ozdulger A, Bastürk M, Degirmenci U, Kanik A, Cinel I. Inhibition of poly(ADP-ribose) polymerase attenuates lung tissue damage after hind limb ischemia-reperfusion in rats. Pharmacol Res 2005; 51: 453-462.

16. Wei Chen Y, Hui Li C, Zhang AQ, Yang SZ, Zhang WZ, Dong JH. Preserving hepatic artery flow during portal triad blood inflow occlusion reduces liver ischemia-reperfusion injury in rats. Surg Res 2011; 1: 7.

17. Chattopadhyay P, Verma N, Verma A, Kamboj T, Khan NA, Wahi AK. L-arginine protects from pringle manoeuvere of ischemia-reperfusion induced liver injury. Biol Pharm Bull. 2008; 31: 890-892.

18. Ota S, Nakamura K, Yazawa T, Kawaguchi Y, Baba Y, Kitaoka R, Morimura N, Goto T, Yamada Y, Kurahashi K. High tidal volume ventilation induces lung injury after hepatic ischemia-reperfusion. Am J Physiol Lung Cell Mol Physiol 2007; 292: L625-631.

19. Schmidt R, Tritschler E, Hoetzel A, Loop T, Humar M, Halverscheid L, Geiger KK, Pannen BH. Heme oxygenase-1 induction by the clinically used anesthetic isoflurane protects rat livers from ischemia/reperfusion injury. Ann Surg 2007; 245: 931-942.

20. Arab HA, Sasani F, Rafiee MH, Fatemi A, Javaheri A. Histological and biochemical alterations in early-stage lobar ischemia-reperfusion in rat liver. World J Gastroenterol 2009; 15: 1951-1957.

21. Atalla SL, Toledo-Perevra IH, Mackenzie GH, Caderna JP. Influence of oxygen-derived free radical scavengers on ischemic livers. Transplantation 1985; 40: 584-590.

22. Song SW, Guo KJ, Shi R, Cheng Y, Liu YF. Pretreatment with calcitonin gene-related peptide attenuates hepatic ischemia/reperfusion injury in rats. Transplant Proc 2009; 41: 1493-1498.

23. Singh K, Khanna AK, Visen PKS, Chander R. Protective effect of ellagic acid on t-butyl hydroperoxide induced lipid peroxidation in isolated hepatocytes. Indian J Exp Biol 1999; 37: 939-940. 
24. Thulstrup PW, Thormann T., Spanget-Larsen J., Bisgaard HC. Interaction between ellagic acid and calf thymus DNA studied with flow linear dichroism UV-VIS spectroscopy. Biochem Biophys Res Commun 199; 265: 416-442.

25. Girish C, Koner BC, Jayanthi S, Rao Kr, Rajesh B, Pradhan SC. Hepatoprotective activity of picroliv,curcumin and ellagic acid compared to silymarin on paracetamol induced liver toxicity in mice. Fundamental Clin Pharmacol 2009; 23: 735-745.

26. Moussavian MR, Scheuer C, Schmidt M, Kollmar O, Wagner M, von Heesen M, Schilling MK, Menger MD. Multidrug donor preconditioning prevents cold liver preservation and reperfusion injury. Langenbecks Arch Surg 2011; 396: 231-241.

27. Takayama F, Egashira T, Kudo Y, Yamanaka Y. Chemiluminescence-HPLC assay of phosphatidylcholine hydroperoxide generated by ischemia-reperfusion in the liver of rats. Biochem Pharmacol 1992; 44 : 2412-2414.

28. Tarpey MM, Wink DA, Grisham MB. Methods for detection of reactive metabolites of oxygen and nitrogen: in vitro and in vivo considerations. Am J Physiol Regul Integr Comp Physiol 2004; 286: R431-444.

29. Hwang JM, Cho JS, Kim TH, Lee YI. Ellagic acid protects hepatocytes from damage by inhibiting mitochondrial production of reactive oxygen species. Biomed Pharmacother 2010; 64: 264-270.

30. Lee HT, Park SW, Kim M, D'Agati VD. Acute kidney injury after hepatic ischemia and reperfusion injury in mice. Lab Invest 2009; 89: 196-208.
31. Park SW, Kim M, Brown KM, D'Agati VD, Lee HT. Paneth cellderived interleukin-17A causes multiorgan dysfunction after hepatic ischemia and reperfusion injury. Hepatology 2011; 53: 1662-1675.

32. Park SW, Chen SW, Kim M, D'Agati VD, Lee HT. Human activated protein $\mathrm{C}$ attenuates both hepatic and renal injury caused by hepatic ischemia and reperfusion injury in mice. Kidney Int 2009; 76: 739-750.

33. Davis CL, Gonwa TA, Wilkinson AH. Pathophysiology of renal disease associated with liver disorders: implications for liver transplantation. Part I. Liver Transpl 2002; 8: 91-109.

34. Polat C, Tokyol C, Kahraman A, Sabuncuoglu B, Yilmaz S. The effects of desferrioxamine and quercetin on hepatic ischemia-reperfusion induced renal disturbance. Prostaglandins Leukot Essent Fatty Acids 2006; 74: 379-383.

35. Miranda LE, Capellini VK, Reis GS, Celotto AC, Carlotti CG Jr, Evora PR. Effects of partial liver ischemia followed by global liver reperfusion on the remote tissue expression of nitric oxide synthase: lungs and kidneys. Transplant Proc 2010; 42: 1557-1562.

36. Yoshidome H, Kato A, Edwards MJ, Lentsch AB. Interleukin-10 inhibits pulmonary NF-kappaB activation and lung injury induced by hepatic ischemia-reperfusion. Am J Physiol 1999; 77: L919-923.

37. Patel S, Pachter HL, Yee H, Schwartz JD, Marcus SG, Shamamian P. Topical hepatic hypothermia attenuates pulmonary injury after hepatic ischemia and reperfusion. J Am Coll Surg 2000; 191: 650-656.

Received June 18, 2011. Accepted February 14, 2012. 